University of South Carolina

Scholar Commons

3-12-2014

\title{
Lorentz Violation in Fermion-Antifermion Decays of Spinless Particles
}

Brett David Altschul

altschul@mailbox.sc.edu

Follow this and additional works at: https://scholarcommons.sc.edu/phys_facpub

Part of the Physics Commons

\section{Publication Info}

Physical Review D, Volume 89, Issue 11, 2014.

(C) 2014 American Physical Society

This Article is brought to you by the Physics and Astronomy, Department of at Scholar Commons. It has been accepted for inclusion in Faculty Publications by an authorized administrator of Scholar Commons. For more information, please contact digres@mailbox.sc.edu. 


\title{
Lorentz Violation in Fermion-Antifermion Decays of Spinless Particles
}

\author{
Brett Altschul] \\ Department of Physics and Astronomy \\ University of South Carolina \\ Columbia, SC 29208
}

\begin{abstract}
If Lorentz and CPT violation exist, they could affect the decays of scalar and pseudoscalar particles. For a decay into a fermion and an antifermion (not necessarily of the same mass), both the total decay rate and the outgoing particle distribution may be modified, through interference between the conventional decay mechanism and a separate Lorentz-violating mechanism. The modifications are sensitive to forms of Lorentz violation that are otherwise rather difficult to study, since at tree level they do not affect particle propagation, but only interaction vertices. Using existing experimental data on charged pion decay, it is possible to constrain three parameters in the modified pionmuon-neutrino coupling at better than the $10^{-9}$ level; these are the first bounds on these quantities.
\end{abstract}

\footnotetext{
${ }^{1}$ baltschu@physics.sc.edu
} 


\section{Introduction}

There is currently quite a bit of interest in the possibility that Lorentz and CPT symmetries might not be exact in nature. At this point, this is strictly a theoretical possibility. No compelling evidence has been found that these symmetries do not hold exactly. However, if such a discovery were made, it would obviously be of the highest importance and would provide concrete evidence for new physics with completely novel behavior.

Both the standard model and general relativity are invariant under Lorentz symmetry and CPT, but deviations from these symmetries involving standard model quanta can be described using the same kind of machinery of effective field theory that is used in the description of the standard model itself. The general effective field theory that can be used to describe such effects is called the standard model extension (SME). The SME contains all possible translation-invariant but Lorentz-violating operators that could be constructed out of known standard model fields. These operators break Lorentz symmetry when they have residual tensor indices. In the Lagrangian, these indices are contracted with tensor-valued coefficients, which describe preferred vectors and tensors [1, 2]. If Lorentz symmetry is spontaneously broken, the preferred background tensors are related to the vacuum expectation values of vector- and tensor-valued dynamical fields.

A particular restricted version of the SME has become the standard framework for parameterizing experimental Lorentz and CPT tests. This is the minimal SME, which contains only local, gauge-invariant, renormalizable operators. With these restrictions, the minimal SME has only a finite number of undetermined parameters, and radiative corrections are calculable; the theory is thus suitable for making orderly comparisons of the results of different experiments. Important tests of spatial isotropy, boost invariance, and CPT symmetries have included studies with matter-antimatter asymmetries for trapped charged particles [3, 4, 5] and bound state systems [6, 7], measurements of muon properties [8, 9], analyses of the behavior of spin-polarized matter [10], frequency standard comparisons [11, 12, 13, 14], Michelson-Morley experiments with cryogenic resonators [15, 16, 17], measurements of neutral meson oscillations [18, 19, 20, 21, 22], polarization measurements on the light from cosmological sources [23, 24], high-energy astrophysical tests [25, 26, 27, 28, precision tests of gravity [29, 30], and others. The best current constraints based on these experiments are collected in [31.

The quantum electrodynamics sectors of the SME has received the most attention, both theoretically and experimentally. In contrast, the least studied sector is is the scalar sector. Fundamental scalars have only recently been observed experimentally, but there are also many composite pseudoscalar particles in the standard model. In addition to the fundamental Higgs, spin-0 mesons may have Lorentz-violating interactions that could be described using the minimal SME formalism. Lorentz-violating Yukawa-like interactions are a particularly rich area; there are many possible interactions, some of which may be quite challenging to constrain. This paper will look at the behavior of a particularly

natural process involving scalars - the production of a fermion-antifermion pair in scalar 
decay. This can have particular relevance in upcoming studies of Lorentz invariance in the Higgs sector, since the Higgs should have an intense top quark decay mode. We shall also look specifically at the decays of pseudoscalar mesons, and it will be possible to place strong constraints on three pion SME coefficients which have never been studied before.

This paper is organized as follows. Section 2 introduces the minimal SME Lagrange density that describes the scalar and spinor fields, as well as their Lorentz-violating interactions. In section 3, we look at several observables - the Lorentz-violating modifications to the decay rate for a scalar particle decaying into a fermion-antifermion pair and the spin structure of the decay when the daughter particles are ultrarelativistic. In section 4 , we shall turn to the problem of charged pion decay, looking at the possibility of interference between the usual weak mechanism for the decay and a novel Lorentz-violating mechanism. It turns out that existing experimental data can provide very strong constraints on some of the types of Lorentz violation that might be involved. Our conclusions and final discussion are given in section 5 .

\section{Lorentz Violation in Scalar-Spinor Interactions}

The coupling of bosonic and fermionic fields is more complicated in a scalar theory than in a gauge theory. In either case, there can be various Lorentz-violating modifications to the boson-fermion vertex. However, in a gauge theory, the renormalizable couplings are completely determined by the Lorentz violation in the pure fermion sector. This is a consequence of gauge invariance, in the form of minimal coupling; the gauge field must couple to the current, which is determined by the way the fermions propagate. There is no such straightforward invariance requirement in a Yukawa theory, and there exist separate Lorentz-violation coefficients that can only be observed in processes involving fermion-boson interaction vertices. Gauge invariance may, however, still restrict which fields may be coupled together, including limiting the chiralities of the spinor fields.

The most prototypical particle interaction process at high energies is pair creation. In a theory with massive bosons, this can take the form of a decay process. The fermion and antifermion produced in the decay may be antiparticles - thus with equal masses - or they may be associated with different fields, so the decay involves a net flavor change.

In our analysis of this pair production process, we shall consider a Lorentz-violating Yukawa theory, with a minimal SME form. With a single fermion field, the most general Lorentz-violating Lagrange density is

$$
\mathcal{L}_{f}=\bar{\psi}\left(i \Gamma^{\mu} \partial_{\mu}-M\right) \psi,
$$

where the purely fermionic Lorentz violation enters through the quantities

$$
\begin{aligned}
\Gamma^{\mu} & =\gamma^{\mu}+\Gamma_{1}^{\mu}=\gamma^{\mu}+c^{\nu \mu} \gamma_{\nu}+d^{\nu \mu} \gamma_{5} \gamma_{\nu}+e^{\mu}+i f^{\mu} \gamma_{5}+\frac{1}{2} g^{\lambda \nu \mu} \sigma_{\lambda \nu} \\
M & =m+i m_{5} \gamma_{5}+M_{1}=m+i m_{5} \gamma_{5}+a^{\mu} \gamma_{\mu}+b^{\mu} \gamma_{5} \gamma_{\mu}+\frac{1}{2} H^{\mu \nu} \sigma_{\mu \nu} .
\end{aligned}
$$


These terms exhaust all the possible Lorentz structures that can be constructed in the minimal SME. The $\Gamma$ coefficients are dimensionless, while the $M$ coefficients have dimension (mass) ${ }^{1}$. A number of the coefficients, such as $m_{5}, a$, and $f$, can actually be eliminated from the theory, through a redefinition of the fermion field [32, 33]. On the other hand, if more than a single fermion species is present in the theory, there may be extra terms with these same structures that mix the fermion fields.

However, Lorentz violation for fermions is a relatively well explored and studied topic. Many efforts, experimental and theoretical, have been made to constrain and understand the $M$ and $\Gamma$ coefficients. We shall only work to first order in the Lorentz violation, so the contributions from various SME coefficients may be determined separately and simply added together. We shall therefore neglect the purely fermionic coefficients and focus on the Lorentz violation that is intrinsic to the scalar sector.

So we shall now introduce a scalar field $\phi$. With this $\phi$, the most general boson-sector Lagrange density with an unbroken $\phi \rightarrow-\phi$ symmetry is

$$
\mathcal{L}=\frac{1}{2}\left(\partial^{\mu} \phi\right)\left(\partial_{\mu} \phi\right)+\frac{1}{2} K^{\mu \nu}\left(\partial_{\nu} \phi\right)\left(\partial_{\mu} \phi\right)-\frac{1}{2} \mu^{2} \phi^{2}-\frac{\lambda}{4 !} \phi^{4}-\bar{\psi}_{a} G_{a b} \psi_{b} \phi .
$$

The tensor $K^{\mu \nu}=K^{\nu \mu}$ plays the same role as $c^{\nu \mu}$ in the fermion sector, and it is the only kind of renormalizable Lorentz violation that can be constructed with a single real scalar field. Like $c, K$ can affect the kinematics for a fermion-antifermion decay process, but $K$ does not affect the matrix element (at tree level). Moreover, coefficients of this general type can be interchanged through coordinate transformations (although some care must be taken to ensure that the final observables are considered in the correct coordinates). So the effects of $K$, like those of $c$ will be neglected here, and we shall concentrate instead on the forms of Lorentz violation described by $G$.

In a gauge theory, any renormalizable Lorentz violation in the boson-fermion coupling is controlled by the same $\Gamma$ coefficients as appear in the pure fermion sector. However, in a scalar field theory, the $G$ that appears in the coupling is independent of the parameters appearing elsewhere in the theory. The form of $G_{a b}$ is

$$
G=g+i g^{\prime} \gamma_{5}+G_{1}=g+i g^{\prime} \gamma_{5}+I^{\mu} \gamma_{\mu}+J^{\mu} \gamma_{5} \gamma_{\mu}+\frac{1}{2} L^{\mu \nu} \sigma_{\mu \nu}
$$

Each of these terms carries the fermion species indices $a$ and $b$. The $a \neq b$ terms generate flavor-changing interactions. (A real $G_{a b}$ must be symmetric in these indices in order to maintain a hermitian Lagrangian; the imaginary part of $G_{a b}$ must likewise be antisymmetric in the flavor indices.) The structures of the various terms contributing to $G$ mirror those seen in $M$. The $g$ and $g^{\prime}$ terms are the Lorentz-invariant scalar and pseudoscalar Yukawa couplings, while the remaining terms are Lorentz violating. All these coefficients are dimensionless, and the tensor term $L^{\mu \nu}$ is naturally antisymmetric. The vector and pseudovector terms $I$ and $J$ break CPT as well as Lorentz invariance, but $L$ does not.

The existence of these terms was noted in the early descriptions of the SME, but very little attention was paid to their rich structure until recently [34, 35, 36]. However, 
with the recent observation of the standard model Higgs boson at the Large Hadron Collider, the importance of fundamental scalars and their interactions with other species has become a central topic in particle physics.

\section{Scalar Boson Decay Behavior}

We shall now look at how the Lorentz-violating contributions to $G$ can affect the decay of a spinless scalar into a fermion and an antifermion - not necessarily with the same mass. The simplest observable in this case is the particle's lifetime; however, we shall see that this turns out to be insensitive to the majority of the coefficients. The rest frame lifetime of the scalar particle depends on $|\mathcal{M}|^{2}$ (where $\mathcal{M}$ is the invariant matrix element for the decay), summed over the outgoing particle spins and integrated over their directions. Of all the Lorentz-violating terms that may appear in the scalar-spinor coupling, only the two terms $I^{0}$ and $J^{0}$ actually influence the final rate. Purely anisotropic terms will not affect the total decay rate, because of a cancellation between different decay channels. In the absence of Lorentz violation, the decay of a scalar particle at rest is necessarily isotropic. A pure anisotropy term in the decay rate, such as one proportional to $I^{k}$, will increase the likelihood of certain decay channels - through a dependence on, for example, $I^{k} p_{k}$ (where $\vec{p}$ is one of the daughter particle momenta). However, decay channels with different momenta will have their importances correspondingly curtailed, leaving the net decay rate unchanged.

The decay rate depends on the quantity

$$
\mathcal{M}=\bar{u}_{1}\left(p_{1}\right) G_{12} v_{2}\left(p_{2}\right) .
$$

This represents the amplitude for the scalar to decay into a fermion with momentum $p_{1}$ and an antifermion with momentum $p_{2}$. Squaring this gives

$$
|\mathcal{M}|^{2}=\bar{u}_{1}\left(p_{1}\right) G_{12} v_{2}\left(p_{2}\right) \bar{v}_{2}\left(p_{2}\right) \bar{G}_{12} u_{1}\left(p_{1}\right)
$$

where $\bar{G}_{a b}=\gamma_{0} G_{a b}^{\dagger} \gamma_{0}$ is the usual Dirac conjugation of $G_{a b}$. The Dirac conjugate simply replaces the $g, g^{\prime}, I, J$, and $L$ coefficients with their complex conjugates; this only affects the coefficients that are off diagonal in flavor space $(a \neq b)$.

Since changes to the fermion sector have been neglected, the sums over the outgoing spins can be evaluated using standard fermion closure relations. This gives (dropping the flavor indices)

$$
\begin{aligned}
\sum_{s_{1}, s_{2}}|\mathcal{M}|^{2}= & \operatorname{tr}\left\{\left(\not \phi_{1}+m_{1}\right)\left(g+i g^{\prime} \gamma_{5}+I^{\mu} \gamma_{\mu}+J^{\mu} \gamma_{5} \gamma_{\mu}+\frac{1}{2} L^{\mu \nu} \sigma_{\mu \nu}\right)\right. \\
& \left.\left(\not p_{2}-m_{2}\right)\left(g^{*}+i g^{\prime *} \gamma_{5}+I^{\mu *} \gamma_{\mu}+J^{\mu *} \gamma_{5} \gamma_{\mu}+\frac{1}{2} L^{\mu \nu *} \sigma_{\mu \nu}\right)\right\} .
\end{aligned}
$$


Expanded to leading order in the $G_{1}$ coefficients, this is

$$
\begin{aligned}
\sum_{s_{1}, s_{2}}|\mathcal{M}|^{2}= & \operatorname{tr}\left\{\left(\not p_{1}+m_{1}\right)\left(g+i g^{\prime} \gamma_{5}\right)\left(\not p_{2}-m_{2}\right)\left(g^{*}+i g^{\prime *} \gamma_{5}\right)\right\} \\
& +\operatorname{tr}\left\{\left(\not p_{1}+m_{1}\right)\left(I^{\mu} \gamma_{\mu}+J^{\mu} \gamma_{5} \gamma_{\mu}+\frac{1}{2} L^{\mu \nu} \sigma_{\mu \nu}\right)\left(\not p_{2}-m_{2}\right)\left(g^{*}+i g^{\prime *} \gamma_{5}\right)\right. \\
& \left.+\left(G \leftrightarrow G^{*}, p_{1} \leftrightarrow p_{2}, m_{1} \leftrightarrow-m_{2}\right)\right\} .
\end{aligned}
$$

The designation $G \leftrightarrow G^{*}$ indicates that the last term in the sum differs from the previous one by the replacement of the $g, g^{\prime}, I, J$, and $L$ coefficients by their complex conjugates (as well as the interchanges of momenta and masses also indicated). Evaluating the traces yields

$$
\begin{aligned}
\sum_{s_{1}, s_{2}}|\mathcal{M}|^{2}= & 4\left(|g|^{2}+\left|g^{\prime}\right|^{2}\right) p_{1} \cdot p_{2}-4\left(|g|^{2}-\left|g^{\prime}\right|^{2}\right) m_{1} m_{2} \\
& -4\left(g^{*} I^{\mu}+g I^{\mu *}\right)\left(m_{2} p_{1 \mu}-m_{1} p_{2 \mu}\right)+4 i\left(g^{\prime *} J^{\mu}-g^{\prime} J^{\mu *}\right)\left(m_{2} p_{1 \mu}-m_{1} p_{2 \mu}\right) \\
& +4 i\left(g^{*} L^{\mu \nu}-g L^{\mu \nu *}+g^{\prime *} \tilde{L}^{\mu \nu}-g^{\prime} \tilde{L}^{\mu \nu *}\right) p_{1 \mu} p_{2 \nu}
\end{aligned}
$$

where $\tilde{L}^{\mu \nu}=\frac{1}{2} \epsilon^{\mu \nu \alpha \beta} L_{\alpha \beta}$ is the dual of $L$.

This expression has a number of noteworthy features. In the rest frame of the decaying scalar, $\vec{p}_{1}=-\vec{p}_{2}$, and when $|\mathcal{M}|^{2}$ is integrated over all decay channels, all the $I$ and $J$ contributions except those related to $I^{0}$ and $J^{0}$ cancel out. Moreover, even these isotropic contributions are nonzero only for flavor-changing decay modes, for which the daughter masses and the time components of $p_{1}$ and $p_{2}$ are unequal.

The energy component of $p_{1}$ is $E_{1}=\frac{\mu}{2}+\frac{m_{1}^{2}}{2 \mu}-\frac{m_{2}^{2}}{2 \mu}$, and the corresponding momentum is $\left|\vec{p}_{1}\right|=\frac{1}{2 \mu} \sqrt{\left(\mu^{2}-m_{1}^{2}-m_{2}^{2}\right)^{2}-4 m_{1}^{2} m_{2}^{2}}$. Consequently, the time component of $m_{2} p_{1 \mu}-$ $m_{1} p_{2 \mu}$

$$
m_{2} E_{1}-m_{1} E_{2}=\left[\frac{\mu}{2}-\frac{\left(m_{1}+m_{2}\right)^{2}}{2 \mu}\right]\left(m_{2}-m_{1}\right) .
$$

The modified decay rate is affected by (11), times the real part of $g^{*} I^{0}$ plus the imaginary part of $g^{\prime *} J^{0}$. These contributions are not invariant under Lorentz boosts, so they could be measured by observing the decay rates of particles with different velocities. This strategy will be discussed in more detail in section 4 .

Since $L$ has no isotropic part, we should expect it to make no contribution at all to the decay rate. This is indeed the case, but the structure of the $L$ terms merits some additional comment. From the structure of the $L$ term in (10), it is evident that only the $L^{0 j}$ and $\tilde{L}^{0 j}$ terms contribute in the scalar rest frame, since $L_{j k}$ is antisymmetric and the spatial momenta $\vec{p}_{1}$ and $\vec{p}_{2}$ are collinear. However, since these six components of $L$ and $\tilde{L}$ actually contain all six $L$ coefficients, the differential decay rate (as a function of the 
direction) can still depend on all the $L$ parameters. The key momentum quantity is

$$
E_{1} p_{2 j}-E_{2} p_{1 j}=-\mu p_{1 j}
$$

which gives a dipolar assymmetry in the decay but clearly does not contribute to the total rate when $\vec{p}_{1}$ is integrated over all angles.

The overall anisotropy of the differential decay rate $d \Gamma / d \Omega$ as a function of the fermion direction $\hat{p}_{1}$ is

$$
\frac{d \Gamma / d \Omega}{(d \Gamma / d \Omega)_{0}}=1+\frac{\sqrt{\left(\mu^{2}-m_{1}^{2}-m_{2}^{2}\right)^{2}-4 m_{1}^{2} m_{2}^{2}}}{|g|^{2}\left[\mu^{2}-\left(m_{1}+m_{2}\right)^{2}\right]+\left|g^{\prime}\right|^{2}\left[\mu^{2}-\left(m_{1}-m_{2}\right)^{2}\right]} W_{j} \hat{p}_{1 j},
$$

where

$$
W_{j}=2 \frac{m_{1}+m_{2}}{\mu}\left(\Re\left\{g^{*} I_{j}\right\}+\Im\left\{g^{\prime *} J_{j}\right\}\right)-\Im\left\{g L_{0 j}+g^{\prime} \tilde{L}_{0 j}\right\} .
$$

The vector $\vec{W}$ governs the anisotropy in a particular rest frame. While it is not possible, using only this decay process, to disentangle the contributions from $I$ from those of $J$ (provided both of the conventional couplings $g$ and $g^{\prime}$ are nonzero), it is possible to separate the $L$ terms, because the $L_{0 j}$ and $\widetilde{L}_{0 j}$ behave differently under Lorentz boosts than do the $I_{j}$ and $J_{j}$.

Finally, we note that for a decay into a fermion and its equal-mass antiparticle, $G_{1}$ makes zero total contribution to the decay rate. Because the coefficients must be real, the $J$ and $L$ terms vanish identically. Because of the equality of the fermion and antifermion masses, the $I^{0}$ term vanishes as well. The only term in (10) that can be nonzero is $16 \mathrm{gm} I^{j} p_{1 j}$. Therefore the only effect on this kind of decay is that the fermion is preferentially emitted along a direction parallel to $\vec{I}$, so the antifermion is preferentially emitted in the antiparallel direction.

So far, we have worked only with the spin-summed decay rate, ignoring the polarizations of the decay products. It is also possible to perform a full analysis, including the spin dependence of the decay amplitudes, at ultrarelativistic energies (large enough that $m_{1}$ and $m_{2}$ may be neglected). This is accomplished by inserting the ultrarelativistic spin projector $\frac{1}{2}\left(1 \pm \gamma_{5}\right)$ into the expression for the matrix element. In this case, the amplitude squared is

$$
\begin{aligned}
|\mathcal{M}|^{2}= & \operatorname{tr}\left\{\frac{1}{2}\left(1+s_{1} \gamma_{5}\right) \not p_{1}\left(g+i g^{\prime} \gamma_{5}+I^{\mu} \gamma_{\mu}+J^{\mu} \gamma_{5} \gamma_{\mu}+\frac{1}{2} L^{\mu \nu} \sigma_{\mu \nu}\right)\right. \\
& \left.\times \frac{1}{2}\left(1-s_{2} \gamma_{5}\right) \not p_{2}\left(g^{*}+i g^{\prime *} \gamma_{5}+I^{\mu *} \gamma_{\mu}+J^{\mu *} \gamma_{5} \gamma_{\mu}+\frac{1}{2} L^{\mu \nu *} \sigma_{\mu \nu}\right)\right\} .
\end{aligned}
$$

In this expression, $s_{1}$ and $s_{2}$ represent the fermion and antifermion helicities, respectively. It is quickly evident that the only Lorentz-violating vertex terms that can contribute are those involving the $L$ coefficients. When the masses are neglected, any term involving a single factor of $I$ or $J$ must necessarily have an odd number of $\gamma$-matrices inside the trace. 
Taking advantage of this fact, and the fact that $g+i g^{\prime} \gamma_{5}+\frac{1}{2} L^{\mu \nu} \sigma_{\mu \nu}$ commutes with $\gamma_{5}$, the $s_{1}$ and $s_{2}$ projectors may be combined into single expressions of the form $\frac{1}{4}(1+$ $\left.s_{1} s_{2}\right)\left(1 \pm s_{1} \gamma_{5}\right)$. Then the expression for $|\mathcal{M}|^{2}$ is

$$
\begin{aligned}
|\mathcal{M}|^{2}= & \frac{1}{4} \operatorname{tr}\left\{\not p_{1}\left(g+i g^{\prime} \gamma_{5}\right) \not p_{2}\left(g^{*}+i g^{\prime *} \gamma_{5}\right)\left(1+s_{1} s_{2}\right)\left(1+s_{1} \gamma_{5}\right)\right\} \\
& +\frac{1}{8} \operatorname{tr}\left\{\not \not_{1} L^{\mu \nu} \sigma_{\mu \nu} \not p_{2}\left(g^{*}+i g^{\prime *} \gamma_{5}\right)\left(1+s_{1} s_{2}\right)\left(1+s_{1} \gamma_{5}\right)\right. \\
& \left.+\not \not_{2} L^{\mu \nu *} \sigma_{\mu \nu} \not p_{1}\left(g+i g^{\prime} \gamma_{5}\right)\left(1+s_{1} s_{2}\right)\left(1-s_{1} \gamma_{5}\right)\right\} .
\end{aligned}
$$

The Lorentz-invariant term in (16) is straightforward. There is an overall factor of $(1+$ $s_{1} s_{2}$ ), which ensures that the outgoing particles have the same helicity, as they should for a state with vanishing total angular momentum. (Although isotropy is broken by the SME interactions, transitions that actually violate angular momentum conservation must have rates that are quadratic in the SME coefficients.)

The rate for the Lorentz-invariant process is just set by the magnitude of the coupling $\left|g+i s_{1} g^{\prime}\right|$ for the outgoing spin state being considered. The $L$-dependent term has exactly the same structure as the $L$ term in (9), with the replacements $g \rightarrow\left(g-i s_{1} g^{\prime}\right)$ and $g^{\prime} \rightarrow\left(i s_{1} g+g^{\prime}\right)$. The trace manipulations can be carried over directly, so the final result for the matrix element squared is

$$
\begin{aligned}
|\mathcal{M}|^{2}= & \left(1+s_{1} s_{2}\right)\left|g+i s_{1} g^{\prime}\right|^{2} p_{1} \cdot p_{2} \\
& +i\left(1+s_{1} s_{2}\right)\left[\left(g^{*}+i s_{1} g^{\prime *}\right) L^{\mu \nu}-\left(g-i s_{1} g^{\prime}\right) L^{\mu \nu *}\right. \\
& \left.+\left(g^{\prime *}-i s_{1} g^{*}\right) \tilde{L}^{\mu \nu}-\left(g^{\prime}+i s_{1} g\right) \tilde{L}^{\mu \nu *}\right] p_{1 \mu} p_{2 \nu} .
\end{aligned}
$$

This expression does not depend on $I$ or $J$, only on $L$. The lack of dependence on these parameters is not surprising. The expression for $|\mathcal{M}|^{2}$ is $\mathcal{O}\left(p_{1} p_{2}\right)$ because of the neglect of the mass terms. There is clearly no structure that is linear in both outgoing momenta but which has only a single Lorentz index to be contracted with $I^{\mu}$ or $J^{\mu}$. This is the ultimate reason why the $I$ and $J$ terms in the trace all involved odd number of Dirac matrices.

\section{Modifications to Pion Decay}

Thus far, we have considered interference between conventional and Lorentz-violating decay mechanisms for a fundamental scalar field. In these scenarios, the Lorentz-symmetric interactions always had a Yukawa-like structure. This kind of analysis is appropriate for studies of Higgs interactions, although Higgs decays are complicated by the additional electroweak couplings of the Higgs sector and by the fact that scalar Higgs decays frequently involve quarks, which are affected by the strong interactions.

In this section, we shall look at how the Lorentz-violating $G_{1}$ couplings can modify a different kind of standard model decay process - the leptonic decay of a meson. For 
definiteness, we shall take the decaying particle to be a pion, although the theoretical analysis could obviously be applied to the weak decays of heavier mesons as well. However, there is actually fairly good data on the boost dependence of the relevant pion decay rate, and this data may be used to constrain the $G_{1}$ coefficients for the pion-muon-neutrino vertex.

The pion is obviously not a fundamental field, and its standard model interaction with the leptons is ultimately mediated by a $W$ boson. However, there is a well-known effective vertex that describes this process, since the virtual $W$ involved is very far off shell. A mechanism with a pion-muon-neutrino $G_{1}$ vertex could produce the same outgoing states, and the two amplitudes for the conventional and Lorentz-violating reaction paths would interfere. In this case, the $G_{1}$ involved should also be seen as representing an effective vertex. The $I, J$, and $L$ involved would be linear combinations of other tensor coefficients associated with the fundamental standard model fields.

The effective interaction Lagrange density for the weak decay of a pion is

$$
\mathcal{L}_{i n t}=-i G_{F} \cos \theta_{C} f_{\pi} \frac{\partial \phi}{\partial x_{\mu}} \bar{\psi}_{1} \gamma_{\mu}\left(1-\gamma_{5}\right) \psi_{\nu}
$$

(plus an obvious hermitian conjugate). The coupling involves the Fermi constant $G_{F}$, the Cabibbo angle $\theta_{C}$, and the pion decay constant $f_{\pi}$. The charged pion $(\phi)$, muon $\left(\psi_{1}\right)$, and massless neutrino $\left(\psi_{2}\right)$ fields all have the Lorentz-invariant free propagation Lagrangians used previously, and we shall consider the same kind of Lorentz-violating $G_{1}$ interaction shown in (41). Because the weak interaction only couples to the left chiral current, Lorentz invariance requires that the standard model coupling between pion, muon, and neutrino have an extra momentum factor to be contracted with the current; this gives the effective interaction dimension (mass) $)^{5}$. However, a Lorentz-violating effective vertex is not so constrained and may be of a lower-dimensional, renormalizable type.

The matrix element squared for the charged pion decay, using both the effective vertex for the $W$ exchange and the novel Lorentz-violating vertex, is (using $\kappa=G_{F} \cos \theta_{C} f_{\pi}$ )

$$
\begin{aligned}
|\mathcal{M}|^{2}= & \left|\bar{u}\left(p_{1}\right)\left[\kappa\left(\not p_{1}+\not p_{2}\right)\left(1-\gamma_{5}\right)+I^{\mu} \gamma_{\mu}+J^{\mu} \gamma_{5} \gamma_{\mu}+\frac{1}{2} L^{\mu \nu} \sigma_{\mu \nu}\right] v\left(p_{2}\right)\right|^{2} \\
\sum_{s_{1}, s_{2}}|\mathcal{M}|^{2}= & \kappa^{2} \operatorname{tr}\left\{\left(\not \not_{1}+m_{1}\right)\left(\not p_{1}+\not p_{2}\right)\left(1-\gamma_{5}\right) \not p_{2}\left(\not p_{1}+\not p_{2}\right)\left(1-\gamma_{5}\right)\right\} \\
& +\kappa \operatorname{tr}\left\{\left(\not \not_{1}+m_{1}\right)\left(\not \not_{1}+\not \not_{2}\right)\left(1-\gamma_{5}\right) \not \not_{2}\left(I^{\mu *} \gamma_{\mu}+J^{\mu *} \gamma_{5} \gamma_{\mu}+\frac{1}{2} L^{\mu \nu *} \sigma_{\mu \nu}\right)\right. \\
& \left.+\left(\not p_{1}+m_{1}\right)\left(I^{\mu} \gamma_{\mu}+J^{\mu} \gamma_{5} \gamma_{\mu}+\frac{1}{2} L^{\mu \nu} \sigma_{\mu \nu}\right) \not \not_{2}\left(\not \not_{1}+\not p_{2}\right)\left(1-\gamma_{5}\right)\right\}
\end{aligned}
$$

The required trace manipulations are very similar to those in the previous calculations. 
The result is (using the on-shell conditions $p_{1}^{2}=m_{1}^{2}=m_{\mu}^{2}$ and $p_{2}^{2}=0$ )

$$
\begin{aligned}
\sum_{s_{1}, s_{2}}|\mathcal{M}|^{2}= & 4 \kappa^{2} m_{1}^{2}\left(p_{1} \cdot p_{2}\right)+4 \kappa m_{1}^{2} p_{2} \cdot\left(I+I^{*}+J+J^{*}\right) \\
& +4 i \kappa m_{1}\left(L^{\mu \nu}-L^{\mu \nu *}\right) p_{1 \mu} p_{2 \nu}+4 \kappa m_{1}\left(\tilde{L}^{\mu \nu}+\tilde{L}^{\mu \nu *}\right) p_{1 \mu} p_{2 \nu} .
\end{aligned}
$$

Note that, of the $I$ and $J$ parameters, only the left-chiral combination $I_{L}^{\mu}=I^{\mu}+J^{\mu}$ enters, since the weak process only involves the left-chiral fermion fields.

The decay rate depends only on the average of $|\mathcal{M}|^{2}$ over all outgoing particle directions. Only those terms which are isotropic in the pion rest frame contribute to this average, so

$$
|\mathcal{M}|_{\text {ave }}^{2}=4 \kappa^{2} m_{1}^{2}\left(p_{1} \cdot p_{2}\right)+4 \kappa m_{1}^{2} E_{2}\left(I_{L}^{0}+I_{L}^{0 *}\right) .
$$

The kinematics of the decay have $E_{1}=\left[\left(1+m_{\mu}^{2} / m_{\pi}^{2}\right) / 2\right] m_{\pi}$ and $\left|\vec{p}_{1}\right|=\left|\vec{p}_{2}\right|=E_{2}=$ $\left[\left(1-m_{\mu}^{2} / m_{\pi}^{2}\right) / 2\right] m_{\pi}$, so that $p_{1} \cdot p_{2}=E_{2} m_{\pi}$. This simplifies the average $|\mathcal{M}|^{2}$ - to which the decay rate $\Gamma$ is directly proportional - so that

$$
\Gamma=\Gamma_{0}\left(1+\frac{I_{L}^{0}+I_{L}^{0 *}}{\kappa m_{\pi}}\right)=\Gamma_{0}\left(1+\frac{I_{L}^{0}+I_{L}^{0 *}}{G_{F} \cos \theta_{C} f_{\pi} m_{\pi}}\right) .
$$

Numerically, this is $\Gamma=\Gamma_{0}\left[1+\left(7.3 \times 10^{6}\right)\left(I_{L}^{0}+I_{L}^{0 *}\right)\right]$, indicating that the Lorentz-violating effect is subject to a large enhancement factor.

The enhancement arises from the interference between decay amplitudes produced by two different dynamical mechanisms. In previously studied scenarios involving Lorentzviolating changes to this decay [37, 38, 39, 40] (as well as most other studies of Lorentz violation in elementary particle processes), the interaction responsible for the process was the conventional, minimally coupled standard model gauge interaction. The Lorentzviolating modifications in previous analyses were essentially changes to the propagation structure for the various fields involved in the decay. These could include Lorentz violation for the parent meson, the daughter leptons, and even the $W$ boson that mediates the weak decay. Such modifications are not just kinematic in nature; they can affect the invariant matrix element $\mathcal{M}$ for the weak decay process, through changes to the propagators for the internal particles and also through the changes to the vector boson vertices that are demanded by gauge invariance.

However, the $G_{1}$ interaction is something else, entirely new, with a strength that is not tied in any way to the strength of the conventional interaction The normal pion decay is rather slow, because the weak interaction is not particularly strong at low energies. So a small $G_{1}$ might still produce a sizable fractional change in $\Gamma$, because $\Gamma_{0}$ is simply quite small to begin with. Even a modestly accurate measurement of the fractional change in $\Gamma$ can thus produce a very strong constraint on the $I_{L}$ parameters; the weakness of the standard model process enhances the sensitivity to a new, Lorentz-violating mechanism.

Although the decay rate depends solely on the component of $I_{L}$ that is isotropic in the pion rest frame, this component $I_{L}^{0}$ does depend strongly on the speed and direction of 
the pion's motion. Experimental bounds on SME parameters are conventionally given in a particular system of Sun-centered celestial equatorial coordinates $(X, Y, Z, T)$ [41]. The origin of the coordinate system is positioned at the center of the Sun. The coordinate $Z$-axis points parallel to the Earth's rotation axis; the $X$-axis points in the direction of the vernal equinox point on the sky; and naturally the $Y$-axis is determined by the right hand rule. The conventional time coordinate is denoted by $T$; however, it is frequently advantageous to use a translated local time coordinate $T_{\oplus}$, selected so that at $T_{\oplus}=0$, the $y$-direction in the laboratory coincides with the $Y$-direction in the reference coordinates.

The coefficient $I_{L}^{0}$ in the pion rest frame is related to the coefficients in the Sun-centered frame by a boost,

$$
I_{L}^{0}=\gamma_{\pi}\left[\left(I_{L}\right)_{T}+\left(\hat{v}_{\pi}\right)_{J}\left(I_{L}\right)_{J}\right]
$$

where $\gamma_{\pi} \gg 1$ and $\hat{v}_{\pi}$ are the Lorentz factor and direction for the pion's motion, respectively. A large value of $\gamma_{\pi}$ provides further enhancement of the sensitivity of the experiment. The reason for this enhancement is that a test of Lorentz symmetry must ultimately involve a comparison of equivalent observables in differently oriented or boosted frames. A large pion speed means that, as the beam direction changes, a collection of very different rest frames is being sampled.

Notably, there are already experimental constraints on how the pion lifetime might depend on the boost of the decaying particle's rest frame. The underlying experimental data was collected by the MINOS experiment and analyzed as part of a search for possible Lorentz-violating neutrino oscillations [42]. There was found to be no evidence of any sidereal oscillations in the number of charged current events in the MINOS near detector. Such oscillations would be a signature of Lorentz violation in the neutrino oscillation probability, but it has been noted [39] that they would also be the signature of Lorentz violation in the pion decay rate. If its lifetime depended on the direction in which a pion is moving, then the overall intensity of the NuMI beam would depend on the orientation of the beam apparatus and thus the time of day.

Note that only the net decay rate is observable via this method. Lorentz violation would generally produce a decay with an anisotropic distribution of decay products in the pion rest frame. However, this anisotropy is effectively washed out by the fact that essentially all the daughter particles are beamed into a narrow pencil of angles along the NuMI beam direction. Moreover, the dependence of the overall NuMI neutrino beam strength on the pion lifetime is a bit more complicated than one might initially anticipate [39]. The length of the pion decay pipe is an important parameter. If the pipe were very long, essentially all the pions in the initial pion beam have time to decay; this would make the neutrino beam intensity almost independent of the pion lifetime. On the other hand, if the pipe were very short, any change in the pion decay rate would be precisely mirrored with the same fractional change in the neutrino beam strength, but the beam so produced would be very weak and the experimental statistics correspondingly poor. For MINOS, where the pipe length was comparable to the mean decay length for the pions involved, a 
change in the observed beam intensity $I$ is related to a change in the pion decay rate $\Gamma$ as

$$
\frac{\Delta I}{I_{0}}=\frac{\Gamma_{0} D / \gamma_{\pi}}{e^{\Gamma_{0} D / \gamma_{\pi}}-1}\left(\frac{\Delta \Gamma}{\Gamma_{0}}\right)=0.31\left(\frac{\Delta \Gamma}{\Gamma_{0}}\right),
$$

where $D$ is the pipe length, and the numerical value was calculated for a typical $6.0 \mathrm{GeV}$ pion.

The geometry of the NuMI beam also determines how the pion boost varies with time. The whole apparatus rotates with the planet, which will produce sidereal oscillations in the key parameter $I_{L}^{0}$. The pion decays occur at colatitude $\chi=42.18^{\circ}$, and the spherical coordinates $(\theta, \phi)$ describe the angle between the beam orientation and the local zenith direction $\left(\theta=93.27^{\circ}\right)$ and the azimuthal angle in the plane of the Earth's surface, measured starting eastward from south $\left(\phi=203.91^{\circ}\right)$. Consequently, the beam direction at a local time $T_{\oplus}=0$ is 43 ]

$$
\begin{aligned}
\hat{v}_{\pi}= & N_{1} \hat{X}+N_{2} \hat{Y}+N_{3} \hat{Z} \\
= & (\cos \chi \sin \theta \cos \phi+\sin \chi \cos \theta) \hat{X}+(\sin \theta \sin \phi) \hat{Y} \\
& +(-\sin \chi \sin \theta \cos \phi+\cos \chi \cos \theta) \hat{Z} \\
= & -0.715 \hat{X}-0.405 \hat{Y}+0.571 \hat{Z} .
\end{aligned}
$$

As the Earth rotates with sidereal frequency $\omega_{\oplus}$, the crucial parameter $I_{L}^{0}$ varies according to

$$
\begin{aligned}
I_{L}^{0}= & \gamma_{\pi}\left\{\left[\left(I_{L}\right)_{T}+N_{3}\left(I_{L}\right)_{Z}\right]+\left[N_{1}\left(I_{L}\right)_{X}+N_{2}\left(I_{L}\right)_{Y}\right] \cos \left(\omega_{\oplus} T_{\oplus}\right)\right. \\
& \left.+\left[-N_{2}\left(I_{L}\right)_{X}+N_{1}\left(I_{L}\right)_{Y}\right] \sin \left(\omega_{\oplus} T_{\oplus}\right)\right\} .
\end{aligned}
$$

Because the rotation of the Earth does not change the $Z$-component of $\hat{v}_{\pi},\left(I_{L}\right)_{Z}$ does not affect the sidereal oscillation amplitude.

Ultimately, the amplitudes of any oscillations in the beam strength are given by the real parts of the sine and cosine coefficients inside the square brackets in (30), times a sensitivity factor

$$
\mathcal{S}=2 \gamma_{\pi}\left(\frac{1}{G_{F} \cos \theta_{C} f_{\pi} m_{\pi}}\right)\left(\frac{\Gamma_{0} D / \gamma_{\pi}}{e^{\Gamma_{0} D / \gamma_{\pi}}-1}\right)=1.9 \times 10^{8} .
$$

The MINOS near detector charge current event rate showed no evidence of oscillations reaching the $3 \sigma$ level of significance (a level that could be seen as indicative of an actual effect). In fact, the levels of signal present in the data were well below the $3 \sigma$ threshold. However, even with no positive evidence for a signal, it was still possible to calculate the the Fast Fourier Transform (FFT) power present in the $\sin \left(\omega_{\oplus} T_{\oplus}\right)$ and $\cos \left(\omega_{\oplus} T_{\oplus}\right)$ modes (as well as in higher harmonics that are not relevant for the present analysis) [42]. The statistical noise that was present in these quadrature modes was characteristic of a fractional $1 \sigma$ dispersion of $1.8 \times 10^{-2}$ in the FFT power. 
Since the MINOS group's analysis looked only at one source of Lorentz violation at a time, we shall take the same approach here. In order that an actual Lorentz-violating $\Re\left\{\left(I_{L}\right)_{J}\right\}$ (for $J=X$ or $Y$ ) should go undetected amidst the noise in the data, the quantity $\left|\mathcal{S} N_{1} \Re\left\{\left(I_{L}\right)_{J}\right\}\right|$ would need to be less than the $3 \sigma$ noise level of $5.4 \times 10^{-2}$. This gives us our final bounds on the coefficients involved,

$$
\left|\Re\left\{\left(I_{L}^{\pi \mu \nu}\right)_{J}\right\}\right|<4 \times 10^{-10}
$$

for $J=X, Y$. The superscripts on $I_{L}$ denote that these are coefficients for the pionmuon-neutrino vertex. The results (32) represent two strong constraints on coefficients that have never previously been bounded.

The pion decay rate is also sensitive to violations purely of boost invariance, through $\left(I_{L}\right)_{T}$. However, this requires comparisons of pion lifetimes for particles with different boost factors, and most precise measurements of the lifetime use stopped pions. Assuming that only $\Re\left\{\left(I_{L}\right)_{T}\right\}$ is nonzero and using the same two data points [44, 45] (at $\gamma_{\pi}=1$ and $\gamma_{\pi}=2.44$ ) discussed in [37], the differences in rest frame lifetimes between stopped pions and pions in motion provide the result

$$
\left|\Re\left\{\left(I_{L}^{\pi \mu \nu}\right)_{T}\right\}\right|=(7 \pm 4) \times 10^{-10} .
$$

So the isotropic boost invariance violation term in $I_{L}$ and two of the three anisotropic terms are constrained at similar levels.

\section{Conclusion}

The constraints (32 33) are the first bounds published on any of the $G_{1}$ coefficients of this type. These Yukawa-like Lorentz-violating parameters are quite numerous in the SME, but they have not been extensively studied. It has been established that the $I, J$, and $L$ play roles in the scalar potentials between fermionic particles and in the renormalization group flow of other SME coefficients. However, neither of these observations has been developed in sufficient detail to make it possible to place practical experimental bounds on the $G_{1}$ parameters. Moreover, such bounds would generally be expected to be rather poor, because of their inherently indirect nature. On the other hand, the constraints given here are quite strong. While the MINOS detector data was not particularly sensitive to sidereal oscillations in the neutrino beam strength, the sensitivity to the relevant $I_{L}$ coefficients was enhanced by two important factors. The relativistic motion of the decaying pions added a factor of $\gamma_{\pi}$, and the weakness of the standard model process meant that a small SME contribution could still make a sizable correction to the net decay rate. Measurements of lepton universality, comparing the branching ratios for meson decays into first- versus second-generation leptons, could also provide sensitivity to differences among the $I_{L}$ parameters. 
The pion, of course, is not a fundamental particle, but a composite made from quarks and gluons. In this paper, we have looked at the two-particle decays both of fundamental scalars, with only renormalizable interactions, and composites, with weak interactions of the type (19). While the standard model certainly contains fundamental scalars - which are only beginning to be studied directly - there also exists a profusion of spin-0 species at the hadronic scale. The fields representing these composite particles have Lorentzviolating operators associated with them in effective field theory. The operators that were constrained in section 4 were of this type. However, while constraints on these and other effective parameters for the pion sector [46] are certainly interesting, it would desirable to understand how the coefficients for particles like pions, protons, and neutrons are related to the coefficients for the underlying quark and gluon fields. In fact, understanding the effective coefficients for hadrons is one of the most important outstanding theoretical problems in the SME.

Understanding Yukawa-like Lorentz violating interactions for the fundamental spin-0 fields will also be important in the coming years, as the detailed behavior of the Higgs particle is mapped out. As it becomes possible to test the Lorentz and CPT invariances of the standard model's scalar sector, the $G_{1}$ coefficients should be an important part of that analysis. For the standard model Higgs, the only $G_{1}$ coefficients that can appear are the $L$ coefficients, because the Higgs field is part of a doublet representation of the $S U(2)_{L}$ gauge field and so can only be coupled to another doublet. While searching for Lorentz violation in the Higgs sector may seem like a difficult undertaking, experimental studies of Lorentz invariance for the similarly heavy and short-lived top quark have already been conducted [47].

This paper has laid groundwork for future studies of Lorentz violation involving scalars, by looking at two-body decays mediated by the Lorentz-violating interactions. The decay rates typically depend on the directions in which both the parent and daughter particles are moving, and both CPT-even and CPT-violating signatures have been identified, associated with different coefficients.

\section{References}

[1] D. Colladay, V. A. Kostelecký, Phys. Rev. D 55, 6760 (1997).

[2] D. Colladay, V. A. Kostelecký, Phys. Rev. D 58, 116002 (1998).

[3] R. Bluhm, V. A. Kostelecký, N. Russell, Phys. Rev. Lett. 79, 1432 (1997).

[4] G. Gabrielse, A. Khabbaz, D. S. Hall, C. Heimann, H. Kalinowsky, W. Jhe, Phys. Rev. Lett. 82, 3198 (1999).

[5] H. Dehmelt, R. Mittleman, R. S. Van Dyck, Jr., P. Schwinberg, Phys. Rev. Lett. 83, 4694 (1999). 
[6] R. Bluhm, V. A. Kostelecký, N. Russell, Phys. Rev. Lett. 82, 2254 (1999).

[7] D. F. Phillips, M. A. Humphrey, E. M. Mattison, R. E. Stoner, R. F. C. Vessot, R. L. Walsworth, Phys. Rev. D 63, 111101(R) (2001).

[8] R. Bluhm, V. A. Kostelecký, C. D. Lane, Phys. Rev. Lett. 84, 1098 (2000).

[9] V. W. Hughes, et al., Phys. Rev. Lett. 87, 111804 (2001).

[10] B. R. Heckel, E. G. Adelberger, C. E. Cramer, T. S. Cook, S. Schlamminger, U. Schmidt, Phys. Rev. D 78, 092006 (2008).

[11] C. J. Berglund, L. R. Hunter, D. Krause, Jr., E. O. Prigge, M. S. Ronfeldt, S. K. Lamoreaux, Phys. Rev. Lett. 75, 1879 (1995).

[12] V. A. Kostelecký, C. D. Lane, Phys. Rev. D 60, 116010 (1999).

[13] D. Bear, R. E. Stoner, R. L. Walsworth, V. A. Kostelecký, C. D. Lane, Phys. Rev. Lett. 85, 5038 (2000).

[14] P. Wolf, F. Chapelet, S. Bize, A. Clairon, Phys. Rev. Lett. 96, 060801 (2006).

[15] H. Müller, et al., Phys. Rev. Lett. 99, 050401 (2007).

[16] S. Herrmann, et al., Phys. Rev. D 80, 105011 (2009).

[17] Ch. Eisele, A. Yu. Nevsky, S. Schiller, Phys. Rev. Lett. 103, 090401 (2009).

[18] V. A. Kostelecký, Phys. Rev. D 61, 016002 (1999).

[19] Y. B. Hsiung, Nucl. Phys. Proc. Suppl. 86, 312 (2000).

[20] K. Abe et al. (Belle Collaboration), Phys. Rev. Lett. 86, 3228 (2001).

[21] J. M. Link et al., Phys. Lett. B 556, 7 (2003).

[22] B. Aubert et al. (BABAR Collaboration), Phys. Rev. Lett. 96, 251802 (2006).

[23] V. A. Kostelecký, M. Mewes, Phys. Rev. Lett. 97, 140401 (2006).

[24] V. A. Kostelecký, M. Mewes, Phys. Rev. Lett. 99, 011601 (2007).

[25] F. W. Stecker, S. L. Glashow, Astropart. Phys. 16, 97 (2001).

[26] T. Jacobson, S. Liberati, D. Mattingly, Nature 424, 1019 (2003).

[27] B. Altschul, Phys. Rev. Lett. 96, 201101 (2006). 
[28] F. R. Klinkhamer, M. Risse, Phys. Rev. D 77, 016002 (2008); addendum Phys. Rev. D 77, 117901 (2008).

[29] J. B. R. Battat, J. F. Chandler, C. W. Stubbs, Phys. Rev. Lett. 99, 241103 (2007).

[30] H. Müller, S. W. Chiow, S. Herrmann, S. Chu, K.-Y. Chung, Phys. Rev. Lett. 100, 031101 (2008).

[31] V. A. Kostelecký, N. Russell, Rev. Mod. Phys. 83, 11 (2011); updated as arXiv:0801.0287v7.

[32] D. Colladay, P. McDonald, J. Math. Phys. 43, 3554 (2002).

[33] B. Altschul, J. Phys. A 39, 13757 (2006).

[34] A. Ferrero, B. Altschul, Phys. Rev. D 84, 065030 (2011).

[35] B. Altschul, Phys. Rev. D 86, 045008 (2012).

[36] B. Altschul, Phys. Rev. D 87, 045012 (2013).

[37] H. B. Nielsen, I. Picek, Phys. Lett. B 114, 141 (1982).

[38] B. Altschul, Phys. Rev. D 84, 091902(R) (2011).

[39] B. Altschul, Phys. Rev. D 87, 096004 (2013).

[40] B. Altschul, Phys. Rev. D 88, 076015 (2013).

[41] R. Bluhm, V. A. Kostelecký, C. D. Lane, N. Russell, Phys. Rev. D 68, 125008 (2003).

[42] P. Adamson, et al. (MINOS Collaboration), Phys. Rev. Lett. 101, 151601 (2008).

[43] V. A. Kostelecký, M. Mewes, Phys. Rev. D 70, 076002 (2004).

[44] M. E. Nordberg Jr., F. Lobkowicz, R. L. Burman, Phys. Lett. B 24, 594 (1967).

[45] D. S. Ayers, et al., Phys. Rev. D 3, 1051 (1971).

[46] B. Altschul, Phys. Rev. D 77, 105018 (2008).

[47] V. M. Abazov, et al. (D0 Collaboration), Phys. Rev. Lett. 108, 261603 (2012). 